\title{
Characteristics of inflammatory cells in urethral smears from men with non-gonococcal urethritis
}

\author{
M SHAHMANESH \\ From the Department of Genitourinary Medicine, St Thomas's Hospital, London
}

SUMMARY Differential cell counts in inflammatory exudates were undertaken in urethral smears from men with non-gonococcal urethritis (NGU) for the first time, recurrent NGU (presumably reinfection) after a new sexual contact, or persistent NGU (in patients with no recent history of sexual exposure). Patients experiencing first episodes of NGU had significantly higher macrophage counts, both in relative and absolute terms, than the other two groups, regardless of the presence or absence of Chlamydia trachomatis in the urethral culture.

The estimated total inflammatory cell count (ETICC) in the urethral exudate was assessed by counting the number of inflammatory cells in the first voided urine. The ETICC was significantly lower in patients with persistent NGU but no recent history of sexual exposure than in patients with NGU for the first time or with NGU presumably caused by a new infection. The ETICC in all three groups of patients was significantly higher than in controls. A more precise definition of NGU or more reproducible methods of diagnosis may possibly differentiate patients without an infective cause.

The aetiological role of Chlamydia trachomatis in $25-58 \%$ of patients with non-gonococcal urethritis (NGU) is well established. ${ }^{1}$ A further $5 \%$ of cases are probably caused by other identifiable organisms, ${ }^{2}$ but the aetiology of the remainder is not known. Some patients with NGU continue to show symptoms and signs of urethral inflammation despite having received conventional antimicrobial treatment and without having had further sexual exposure. It has been suggested that in some of these patients this is due to an abnormal immune reaction. ' ${ }^{\prime}$ The present study was undertaken to examine the characteristics of the inflammatory exudate from the urethras of patients with NGU. The findings suggest that some patients have persistent NGU not directly caused by reinfection. Differential cell counts made similarly in bronchial expirates have been helpful in elucidating the various aetiologies of interstitial pulmonary disease. ${ }^{3}$

\section{Patients and methods}

I studied 75 heterosexual men aged 17 to 50 (mean 28.8) attending the sexually transmitted disease (STD)

Address for reprints: Dr M Shahmanesh, Department of Genitourinary Medicine, General Hospital, Steelhouse Lane, Birmingham B4 6NH

Accepted for publication 25 June 1988 clinic at St Thomas's Hospital. Nineteen were black, and the remaining 56 were white. I took a full sexual history from each patient and made a complete genitourinary examination. All patients complained of urethral discharge or dysuria, or both, and on examination all were found to have urethral discharge. Patients were included in the study if they had passed urine more than two hours before being examined (mean 4(SD 2.2), range 2-9 hours). From each patient I took a urethral smear using a platinum wire loop, which I passed $1-1.5 \mathrm{~cm}$ into the urethra, smeared evenly on a slide, and Gram stained. The remainder of the specimen was cultured for Neisseria gonorrhoeae. Using cotton tipped wire mounted swabs I took further specimens for culture for $C$ trachomatis from 32 of the patients. ${ }^{4} \mathrm{~A}$ two glass urine test was then performed. NGU was diagnosed when more than 10 leucocytes were seen in 10 high power $(\times 1000$ magnification) fields (HPF). ${ }^{s}$

I studied three groups of patients: men presenting with their first attack of urethritis; men with a documented history of urethritis who presented with recurrent NGU within three weeks of intercourse with a new sexual partner; and men who had had three or more attacks of urethritis in the previous 18 months but no history of sexual exposure in that time. Twelve patients aged 20 to 30 who presented at the STD clinic with no history or signs of urethritis and who were 
found to have fewer than four leucocytes/HPF in their urethral smears were used as controls. Cultures for chlamydiae were performed on four controls.

The study was performed in two parts. The first part contained 46 patients (18 with first NGU, 19 with reinfection, and nine with persistent NGU) from each of whom a second urethral smear was taken, air dried, and stained with May-Grunwald-Giemsa (MGG). A differential inflammatory cell count was performed by examining 200-500 cells without knowing the patient's history. The second smear from 13 of these patients was divided between two slides, the second being fixed in alcohol and stained by Papanicolaou's method.

The second part of the study contained 49 patients (17 with first episode NGU, 21 reinfected, and 11 with persistent NGU). For each of these patients the total number of inflammatory cells in the urethral exudate remaining after the urethral smears had been taken was counted. Twenty of these patients had undergone a second urethral smear for MGG staining. After the urethral smears had been taken, the patients were asked to urinate into two glasses. Urine from the first glass was centrifuged for three minutes at $600 \times \mathrm{g}$, the pellet was resuspended in $1 \mathrm{ml}$ saline, and the cells were counted in a Neubauer chamber. Cell counts were expressed as the total number per urethral exudate. As some of the exudate had been removed by the smears, this figure gives only an estimate of the true number of inflammatory cells in the urethral exudate.

To assess whether the macrophages were capable of phagocytosis, the first sample of urine from four patients with first attack NGU was centrifuged (for three minutes at $600 \times \mathrm{g}$ ) and the pellet washed with $5 \mathrm{ml} \mathrm{RPW} 1460$ medium. This was then centrifuged, resuspended in $1 \mathrm{ml}$ RPW medium and latex particles, and incubated at $37^{\circ} \mathrm{C}$ for 30 minutes. The sample was then centrifuged and the pellet smeared on a glass slide, stained with MGG, and examined under a light microscope for a differential cell count.

\section{STA TISTICAL ANALYSES}

Analysis of variance was performed for all data. Comparisons between the differential cell counts in the three groups of patients were made using the Mann-

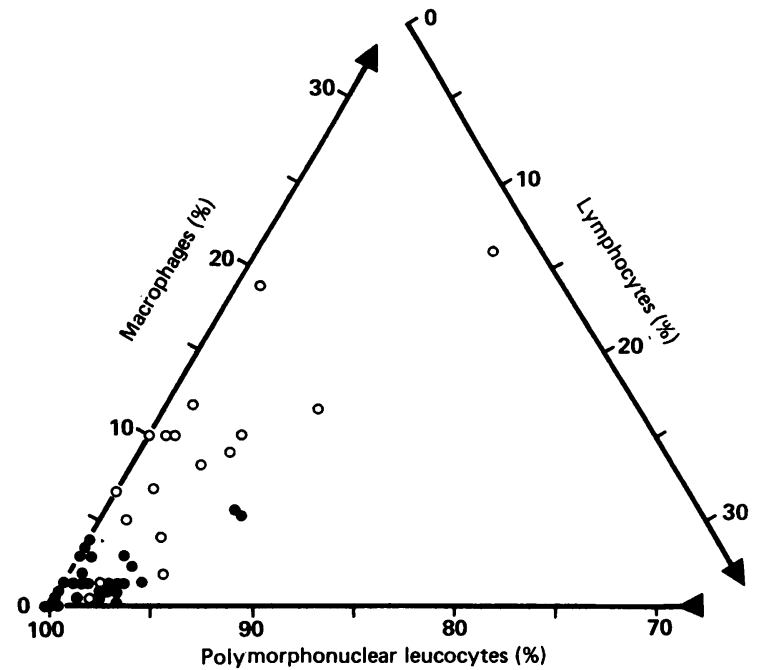

Fig 1 Individual differential cell counts in urethral smears from 17 men with non-gonococcal urethritis (NGU) for the first time (O) and 28 with presumed reinfection or with persistent $N G U$ but no recent history of sexual exposure (O).

Whitney U test, and of the ETICC in urethral exudates using Student's $t$ test on log transformed data.

\section{Results}

Table 1 and fig 1 show results from the first part of the study. Table 1 shows the relative proportion of different inflammatory cells in urethral exudates. Polymorphonuclear leucocytes predominated in the urethral exudates from all three groups. Patients infected with NGU for the first time had a higher percentage of macrophages in their urethral smears than those presumably reinfected after a new sexual contact or who had persistent NGU $(p<0.01)$. No difference was seen in the differential cell profiles between reinfected men and those with persistent NGU. Fig 1 shows the individual differential cell counts. Only two of the 28 patients with presumed reinfection or persistent NGU had more than $5 \%$

Table 1 Percentages of different inflammatory cells in urethral exudates from men with non-gonococcal urethritis (NGU) for the first time, presumed reinfection, or recurrence without known sexual exposure (figures are mean (SD) obtained from May-Grunwald-Giemsa and Papanicolaou stained smears)

\begin{tabular}{lccc}
\hline Cells & $\begin{array}{l}\text { First NGU } \\
(n=18)\end{array}$ & $\begin{array}{l}\text { Reinfection } \\
(n=19)\end{array}$ & $\begin{array}{l}\text { Persistent NGU } \\
(n=9)\end{array}$ \\
\hline Macrophages & $8 \cdot 5(5 \cdot 4)^{*}$ & $2 \cdot 0(1 \cdot 5)$ & $1 \cdot 3(1 \cdot 7)$ \\
Lymphocytes & $2 \cdot 6(2 \cdot 6)$ & $1 \cdot 6(1 \cdot 8)$ & $1 \cdot 6(1.3)$ \\
Polymorphonuclear leucocytes & $89(7 \cdot 0)$ & $96 \cdot 3(2 \cdot 3)$ & $97 \cdot 2(2 \cdot 3)$ \\
\hline
\end{tabular}

*p $<0.01$ compared with both other groups of patients. 


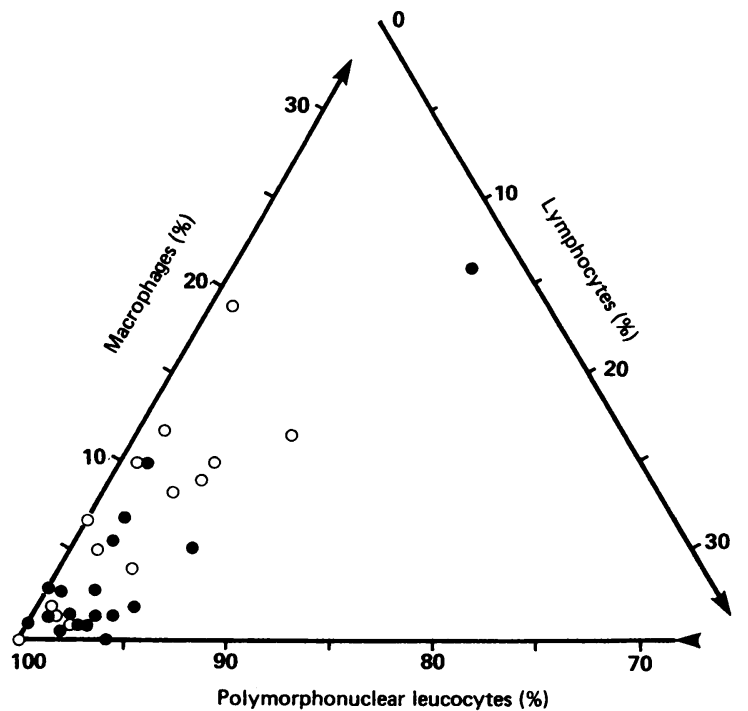

Fig 2 Individual differential cell counts in urethral smears from 32 men with non-gonococcal urethritis (NGU) in relation to the presence $(O, n=14)$ or absence

$(0, n=18)$ of Chlamydia trachomatis in urethral cultures.

macrophages in the stained urethral smears. In contrast, 13 of 17 patients with NGU for the first time had $5 \%$ or more macrophages in the stained urethral smears.

Of the 32 patients for whom cultures for chlamydiae were performed, 14 gave positive results; 10/18 $(55.5 \%)$ of those with first episodes of NGU, and 4/19 $(21 \%)$ of those assumed to be reinfected. None of those with persistent NGU and no controls yielded chlamydiae. Infection with chlamydiae did not affect the differential cell count (fig 2).

The coefficient of variation between MGG stained and Papanicolaou stained samples from the 13 patients for whom both staining techniques were used was $2.6 \%$ for polymorphonuclear leucocytes and $26.7 \%$ for macrophage counts. The figures in table 1 therefore express the mean obtained from both smears from those 13 patients. Macrophages incubated with

Table 2 Estimated total inflammatory cell count (ETICC) in first voided urine samples from patients with $N G U$ for the first time, presumed new infection, or persistent $N G U$ without known sexual exposure, and controls (figures are mean (SEM) numbers of cells $\times 10^{4}$ )

\begin{tabular}{llll}
\hline $\begin{array}{l}\text { First NGU } \\
(n=17)\end{array}$ & $\begin{array}{l}\text { Reinfection } \\
(n=21)\end{array}$ & $\begin{array}{l}\text { Persistent } \text { NGU } \\
(n=11)\end{array}$ & $\begin{array}{l}\text { Controls } \\
(n=12)\end{array}$ \\
\hline $907(218)$ & $344(68)$ & $97(59)$ & $4 \cdot 7(0.9)$ \\
\hline
\end{tabular}

First NGU $v$ persistent NGU, $\mathrm{p}<0.001$; reinfection $v$ persistent NGU, $p<0.001$; all three groups of patients $v$ controls, $p<0.001$; first NGU $v$ reinfection, NS. latex particles were shown to be capable of phagocytosis.

Table 2 shows the estimated total inflammatory cell count (ETICC) in the first voided urine samples from the three groups of patients in the second part of the study. Men with first episodes of NGU and those reinfected had a significantly higher ETICC than those with persistent NGU $(p<0.001)$. The difference between men with first episodes and those reinfected was not significant. The ETICC of each of the three groups of patients with NGU was significantly higher than that of the controls $(p<0.001)$.

A rough index of the absolute macrophage content of the urethral exudate could be assessed in eight men with first episodes of NGU and 13 reinfected men, for whom both a differential cell count and an ETICC in the first voided urine sample was available. Patients with first attack NGU (group I) had more macrophages (mean (SE) 475 (180)) than reinfected men $(33.2(6.8))(p<0.01)$.

\section{Discussion}

This study shows that though the predominant inflammatory cells in all patients with NGU were polymorphonuclear leucocytes, urethral exudates from patients who had developed NGU for the first time contained more macrophages, in relative and absolute terms, than patients who experienced subsequent attacks of NGU. This difference was seen whether chlamydiae were present or absent and was also evident whether the recurrence was caused by reinfection or reinfection could not be documented. Differential inflammatory cell counts of the urethral exudate cannot, therefore, differentiate recurrent NGU caused by reinfection from NGU in which sexual transmission cannot be established.

The differences observed in the macrophage counts between first attack NGU and subsequent attacks of NGU remain to be explained. Though little is known about the pathophysiology of NGU, some information is available regarding the role of macrophages and other effector cells in infection with $C$ trachomatis, ${ }^{6}$ which is the infective organism in $25-58 \%$ of patients infected for the first time. ${ }^{1}$ Alani et al found a lower incidence of chlamydial infection in subsequent attacks, ${ }^{5}$ which is supported by the findings of this report. Ingestion by macrophages seems to play an important part in infections with chlamydiae, ${ }^{78}$ though polymorphonuclear leucocytes and lymphocytes may also contribute to the elimination of the organism.

Macrophages play an important part in the immune response. ${ }^{9}$ They are believed to process antigen before presenting it to lymphocytes as part of their immune function. Macrophages in the urethral exudates of 
patients with NGU for the first time are possibly fulfilling the function of antigen presentation, among other functions.

The inflammatory response, as judged by the total number of inflammatory cells in the urethral exudate, of patients in whom recurrent NGU could not be attributed to sexual transmission was significantly lower than in patients in whom recurrent NGU was caused by a presumed new infection, and significantly higher than in controls (table 2). The diagnosis of NGU in all the patients was made by detecting more than 10 leucocytes/HPF. ${ }^{5}$ The difficulty in reproducing results in urethral smears from men with NGU has, however, been documented..$^{10} \mathrm{~A}$ more strict criterion for the diagnosis of NGU might exclude most patients in whom an infective cause is unlikely, and therefore avoid unnecessary antibiotic treatment of some patients and their sexual partners. Further work relating cell counts in urethral smears to "absolute" cell counts in the urethral exudate may be helpful in refining these criteria. The possibility exists, however, that detecting inflammatory cells in the urethral exudate may not in itself be sufficient evidence of infection.

I thank Drs Nicol Thin and David Barlow for their helpful comments on the paper, Ms E Paul for performing the statistical analysis, and the technicians at the cytology laboratory for their help in preparing the stained specimens.

\section{References}

1 Taylor-Robinson D, Thomas BJ. The role of Chlamydia trachomatis in genital-tract and associated diseases. J Clin Pathol 1980;33:205-33.

2 Hawkins DA, Taylor-Robinson D. Non-gonococcal urethritis in the male. In: Asscher AW, Brumfitt W, eds. Microbial diseases in nephrology. Chichester: John Wiley, 1986:47-67.

3 Crystal RG, Bitterman DB, Rennard SI, Hance AJ, Keogh BA. Interstitial lung disease of unknown cause. Disorders characterised by chronic inflammation of the lower respiratory tract. $N$ Engl J Med 1984;310:154-66, 235-44.

4 Bradbeer CS, Thin RN. Comparison of econazole as a single dose treatment of vaginal candidosis. Genitourin Med 1985;61:396-8.

5 Alani MD, Darougar S, Burns MacD, Thin RN, Dunn H. Isolation of Chlamydia trachomatis from the male urethra. British Journal of Venereal Diseases 1977;53:88-92.

6 Monnickendam MA, Pearce JH. Immune responses and chlamydia infections. Br Med Bull 1983;39:187-93.

7 Wyrick PB, Brownridge EA. Growth of Chlamydia psittaci in macrophages. Infect Immun 1978;19:1054-60.

8 Lammert J. Cytotoxic cells induced after Chlamydia psittaci infection in mice. Infect Immun 1982;35:1011-7.

9 Johnston RB. Current concepts: immunology. Monocytes and macrophages. $N$ Engl J Med 1988;318:747-52.

10 Wilcox JR, Adler MW, Belsey EM. Observer variation in the interpretation of Gram-stained urethral smears: implications for the diagnosis of non-specific urethritis. British Journal of Venereal Diseases 1981;57:134-6. 